\title{
Community Psychiatry, Community Mental Health Services or Primary Care Psychiatry?
}

\section{E K Rodrigo}

Community Psychiatry implies a narrower brief than Community Mental Health Services or Care; the former implies provision of treatment for mentally ill people, most commonly the severely mentally ill. A World Health Organization (WHO) Regional Workshop on 'Developing mental health services' in South East Asia in 2006 concluded "Community mental health services should meet all the mental health needs of the community including mental health promotion, prevention of mental illness, psychosocial needs of the community, needs of special groups (adolescents, elderly and refugees) and prevention of harm from substance abuse" (1). As the Director of Mental Health, Sri Lanka, and a consultant psychiatrist from the National Institute of Mental Health participated in this workshop, it is presumed that Sri Lanka agreed with this definition.

Thornicroft et al. appear to agree with the WHO definition when they state that Community Mental Health Care "comprises of the principles and practices needed to promote mental health for a local population by: (a) addressing population needs in ways that are accessible and acceptable: (b) building on the goals and strengths of people who experience mental illnesses: (c) promoting a wide network of supports, services and resources of adequate capacity: (d) emphasising services that are both evidence based and recovery oriented" (2).

However, critiques of community psychiatry as practiced in the developed countries claim that this is not an applicable framework for developing countries. Farooq and Minhas proposed abandoning the term community psychiatry in favour of Primary Care Psychiatry (3). A similar comment was made by Jane and Jadhav (4). Similarly, the World Psychiatric Association (WPA) Lancet Psychiatry Commission on the Future of Psychiatry, states that there is no universal public health service delivery, which will work in all countries (5). This commission also observes that models of care provision are dependent on the availability and type of human and financial resources, organisation of its health care systems, and specific mental health needs of the people in a given country.

Does Sri Lanka have the necessary resources to provide such a service? If the policy decision has already been made, who, where and how will these services be provided?
Does Sri Lanka have the data regarding mental health needs in the population? We know that Sri Lanka has a relatively high suicide rate and domestic violence, and child abuse is not uncommon. Sri Lanka is also emerging from an ethnic armed conflict that lasted for more than three decades. This was compounded by two insurrections that occurred in the early seventies and late eighties. Sri Lanka was also unfortunate to experience a tsunami with devastating effects. More recently there have been landslides and floods as well. What are the mental health consequences of such disasters - man made and natural? Another major factor to be considered, that is likely to significantly impact on mental health, particularly of children, is the migration of a large number of females seeking overseas employment.

Additionally, social determinants of mental health need to be considered when planning these services. These core social determinants of mental health are listed by Compton and Shim as, discrimination and social exclusion, adverse early life experiences, poor education, unemployment, underemployment and job insecurity, income inequality, poverty and neighbourhood deprivation, food insecurity; adverse features of the build environment and poor access to health care (6). Man made and natural disasters of the past few decades have compounded the effects of these social determinants in Sri Lanka.

The WHO too proposes a multi level framework for understanding social determinants of mental illness (7). In Sri Lanka at present, psychiatric services are mostly hospital based; about a hundred psychiatrists provide psychiatric care, mostly through General Hospital and Base Hospital units and affiliated outpatient clinics. A majority of the psychiatrists working in the public sector also provide additional services in the private sector. These combined services correspond to one half of the fourth tier described in the WHO Service Organization Pyramid for optimal provision of services for mental health (8). However, the community mental health service component of this fourth tier is lacking in Sri Lanka. And the third tier of the WHO Pyramid model, which represents primary care mental health services, is at best rudimentary.

A third of the psychiatrists in the public sector in Sri Lanka provide only an outpatient service, as they do not have inpatient units. On one hand this maybe considered 
an advantage, as Sri Lanka does not have to dismantle large institutional mental health care systems, unlike in the high-income countries. A possible development pathway is to support these psychiatrists who do not have inpatient units, is to develop community mental health services in collaboration with the existing primary health care network. Psychiatrists who are at present providing only an outpatient service could develop a service similar to the 'Trieste Model' where 95\% of the resources are utilized in the community, enhancing autonomy of patients, strengthening families and organising alternative settings of care with minimal use of hospital beds (9).

Sri Lanka is fortunate in having an extensive island wide primary health care network. Medical officers of health, public heath nurses, midwives and heath inspectors provide both preventive and curative services. Already several programmes have trained primary care clinicians in mental health care. However, such training programmes have not been consistent or island wide.

Like many low and middle-income countries, in the near future Sri Lanka is unlikely to have the required number of psychiatrists (per population). It is physically impossible for the existing number of psychiatrists to see and treat all the persons requiring mental health care. The same applies for clinical psychologists, psychiatric nurses, social workers and occupational therapists in the country. Therefore, Sri Lanka will have to consider provision of community mental health services through non-specialised staff. This would mean adequate training of non-specialised staff members and providing them with adequate guidance and supervision. This would require a review of the role of the psychiatrist - more as a trainer and a supervisor rather than only as a therapist. This would mean that the training curriculum of psychiatrists should be modified, to enable them to work not only in the hospital setting, but in the primary health care setting as well. The psychiatrist would then play a key role in the development of the capacity of primary health care workers to detect and treat people with mental illness.

If such training can be provided throughout the island on an on-going basis, do these primary health care clinicians have the capacity to take over this additional mental health care load? Or is it an opportune time for Sri Lanka to recruit and train non-specialist, community mental health care workers?

Already, some of the community mental health care work in Sri Lanka is being carried out by Non Governmental Organisations (NGOs): 'Sahanaya' in the Western Province, 'Nivahana' in the Central Province, 'Shantiham' in the Northern Province, 'Nest' which provides an island wide service and the Health Promotion Foundation, which is working towards empowering families, particularly women and communities in several villages.
These organisations have provided training to primary health care clinicians, teachers, social service workers and others, while some have provided recovery and rehabilitation services to some of the patients discharged from psychiatric units.

As Sri Lanka is likely to experience a paucity of experienced mental health clinicians, the most pragmatic way forward is for the psychiatrists to collaborate with the primary health care network, NGOs and other relevant stakeholders and service providers to deliver mental health care services to mentally ill persons, and to promote mental health. Legislators, policy makers, national and provincial ministries of health, psychiatrists, other stakeholders and the general public need to determine what is required, what is possible and what needs to be developed. As a group, psychiatrists also need to advocate for the mentally ill people, particularly to ameliorate negative social determinants of mental health; and they need to educate and train themselves to promote mental health and wellbeing. Sri Lanka is fortunate to have accessible, island wide health and education services at primary, secondary and tertiary levels, which can be utilised to develop mental health promotion initiatives and interventions. Unlike many low and middle-income countries, Sri Lanka possesses services and structures that can be harnessed to develop an effective primary mental health care service to its people.

\section{Disclosure statement}

None declared

E K Rodrigo, Former Senior Lecturer, Department of Psychiatry, Faculty of Medicine, Peradeniya, Sri Lanka and Senior Staff Specialist, City Mental Health Unit, Canberra, ACT, Australia.

Email: Kalyana.Rodrigo@act.gov.au

\section{References}

1. World Health Organization (WHO). Developing community mental health services; Report of the regional workshop Bangkok, Thailand, 11th -14th December 2006. New Delhi: WHO, 2007.

2. Thornicroft G, Deb T, Henderson C. Community mental health care world wide: current status and further developments. World Psychiatry 2016; 15(3): 276-86.

3. Farooq S, Minhas FA. Community psychiatry in developing countries - a misnomer? Psychiatr Bull 2001;25:226-7. 
4. Jane S, Jadhav S, A Cultural critique of community psychiatry in India. Int J Health Serv 2008; 38(3):561-84.

5. Bhugra D, Tasman A, Pathare S, et al. The WPA-Lancet psychiatry commission on the future of psychiatry. Lancet Psychiatry 2017; 4(10): 775-818.

6. Compton MT, Shim RS. The social determinants of mental health. Washington DC: American Psychiatric Association, 2015.

7. World Health Organisation and Calouste Gulbenkian
Foundation. Social determinants of mental health. Geneva: World Health Organisation, 2014.

8. World Health Organization (WHO). Improving health systems and services for mental health. Geneva: WHO, 2009.

9. Mezzina R. Community mental health care in Trieste and beyond: An "open door-no restraint" system of care for recovery and citizenship. J Nerv Ment Dis. 2014; 202(6): 440-5. 